\title{
Cardiotoxicity following a suicide attempt by Yew Tree Seed Ingestion: A case report
}

\author{
Alexandra Tebbett ${ }^{1}$ and Claire Walton ${ }^{1}$ \\ ${ }^{1}$ South Warwickshire NHS Foundation Trust
}

February 3, 2022

\begin{abstract}
The taxine alkaloids within the seeds, leaves and bark of the Yew Tree can cause death via antagonising cardiac Ca2+ channels. We report the case of a 17-year-old male who presented with severe cardiogenic shock with biventricular block following the consumption of Yew Tree seeds that he had purchased online.
\end{abstract}

Cardiotoxicity following a suicide attempt by Yew Tree Seed Ingestion: A case report

A. Tebbett ${ }^{1}$ and C. Walton ${ }^{2}$

1 Anaesthetic Registrar, South Warwickshire Foundation Trust, Warwick, United Kingdom

2 Critical Care Outreach Nurse, South Warwickshire Foundation Trust, Warwick, United Kingdom

Correspondence to: Dr Tebbett (atebbett@doctors.org.uk)

Abstract :

The taxine alkaloids within the seeds, leaves and bark can of the Yew Tree cause death via antagonising cardiac $\mathrm{Ca} 2+$ channels. We report the case of a 17-year-old male who presented with severe cardiogenic shock with biventricular block following the consumption of Yew Tree seeds that he had purchased online.

Key clinical message:

This method of toxicity was previously unknown to all clinicians involved in his care, but he was treated successfully by following Toxbase guidelines. It was disturbing to learn these seeds can be purchased easily online.

\section{Case Report Article}

Introduction :

The Yew Tree (Taxus Baccata) is an evergreen tree native to Europe. In the UK it is often found in churchyards due to their historic link with immortality and death (1). Another possible reason was to deter farmers from grazing their livestock on church grounds, as the Yew Tree is extremely poisonous (1). The taxine alkaloids contained in the seeds, needles and bark can produce a lethal dose at even small quantities via cardiogenic shock (2). Accidental consumption in non-lethal quantities is common in children (3) but only a few cases of attempting suicide by ingestion have been reported, most frequently with lethal outcomes $(3,4,5)$. Here we discuss the case of a 17-year-old male who presented with acute cardiogenic shock with a ventricular escape rhythm following ingestion of Yew Tree seeds, with a non-lethal outcome.

\section{Case Report:}


A 17-year-old male with a background of asthma took approximately 100g of Yew Tree seeds at around 22:30 one evening in an attempt to end his life. Assessment at the scene revealed bradycardia at 40 beats/minute, hypotension at around $60 / 30 \mathrm{mmHg}$ and hypothermia. His Glasgow Coma Score (GCS) was 15/15. He was treated with IV Hartmann's and 15L oxygen and brought to the nearby district general hospital where he was admitted at 03:08am.

On arrival to the Emergency Department his ECG showed a ventricular rhythm with a prolonged QT (QT $670 \mathrm{~ms}$ / QTc 414ms) (Figure 1), so external pacing pads were applied in preparation. His initial venous blood gas showed a $\mathrm{pH}$ of 7.19 with a HCO3- of 24.8, $\mathrm{BE}$ of -4.8 and lactate of 3.5 (other values largely normal) (Table 1).

Initial peripheral infusions were started: magnesium $1 \mathrm{~g}$, sodium bicarbonate $1.26 \%$ at $83.3 \mathrm{ml} / \mathrm{hr}$, and Dobutamine $250 \mathrm{mg}$ in $500 \mathrm{ml} 5 \%$ dextrose at $20 \mathrm{ml} / \mathrm{hr}$. Dobutamine was chosen over adrenaline due to recommendations from Toxbase that adrenaline can worsen hypotension, possibly due to beta agonist effects (6). Dobutamine was rapidly titrated up to $30 \mathrm{ml} / \mathrm{hr}$ but his bradycardia worsened to 20 beats $/ \mathrm{min}$ and his GCS dropped. External pacing was commenced and restored circulation. Electrical capture was first obtained at $80 \mathrm{~mA}$, but this needed to be titrated up to $120 \mathrm{~mA}$ to sustain mechanical capture.

Once central venous access was established, Isoprenaline $2 \mathrm{mg}$ in $500 \mathrm{mls} 5 \%$ glucose was started at $30 \mathrm{mls} / \mathrm{hr}$. This was increased over the next 5 minutes to $98 \mathrm{ml} / \mathrm{hr}$ to allow for cessation of external pacing. Recommendations from Toxbase to aim for a $\mathrm{pH}$ of 7.5-7.55 (6) were noted, and three boluses of $8.4 \%$ sodium bicarbonate were given which resulted in a $\mathrm{pH}$ of 7.53 at 04:30, 82 minutes since first admission to hospital (Table 1).

On advice from UK NPIS, ECMO was contacted for consideration of transfer, but it was deemed to be too early at this stage of his treatment. The on-call cardiologist successfully inserted a right ventricular temporary pacing wire allowing Isoprenaline to be switched off, showing an underlying rhythm of asystole. Pacing capture was obtained at $0.5 \mathrm{~mA}$ and the rate set to 80 beats/minute, giving a blood pressure of 100/48 mmHg.

The patient was admitted to the Intensive Care Unit where he received a Covid-19 throat swab which triggered an episode of emesis, in which he regurgitated a mixture of whole and semi-digested seeds. Over the next 12 hours he was treated with further sodium bicarbonate, potassium, magnesium and insulin therapy. Dobutamine was switched to an adrenaline infusion which was quickly titrated down and stopped. The threshold for his internal pacing wire increased to $1 \mathrm{~mA}$ but held at that point. A bedside echocardiogram showed no obvious abnormalities besides bilateral pleural effusions.

28 hours after admission he switched into an unpaced broad complex $1^{\text {st }}$ degree block rhythm with a QTc of 525ms. On day three his pacing wire was removed. He was discharged home on day six after seeing psychiatry, with further psychiatry follow-up and an outpatient echocardiogram arranged.

\section{Discussion:}

The yew tree is a common, native poisonous evergreen with bright red-berries that could be appealing to young children (1). If searching through graveyards and harvesting the tree is unappealing, the seeds can be purchased easily online, as happened in this case. Once ingested (and particularly if chewed during ingestion) the taxine alkaloids are absorbed where their main action is on cardiac myocytes, where they primarily antagonise the $\mathrm{Ca} 2+$ channels causing heart block and eventually cardiac arrest (2). It is estimated that $1 \mathrm{~g}$ of yew leaves contain $5 \mathrm{mg}$ of taxines, and the lethal dose can be as low as $3.0 \mathrm{mg} / \mathrm{kg}$ in humans (2).

Previous case studies on Yew Tree poisoning in adults generally outline lethal cardiotoxicity, and provide pessimistic reading whilst awaiting the arrival of a similar patient in the emergency department. With no prior experience in treating taxine alkaloid toxicity, and with a relatively junior on-call night staff in a district general hospital, this case had a more positive outcome. Guidance from Toxbase (6) previously published case reports and the experience of a cardiothoracic nurse working on the critical care outreach team overnight were pertinent to this outcome. 
The decision to use Dobutamine (a B1-agonist) and Isoprenaline (a non-selective beta agonist) over Atropine (an antimuscarinic alkaloid) and Adrenaline (a non-selective alpha and beta agonist) meant the drug administered were more cardio-specific than what may otherwise be used and the aforementioned hypotension as a result of the non-specific actions of adrenaline was not noted. However with hindsight, and having received no ill effects on switching to an adrenaline infusion in the ICU, we would have a lower threshold for using adrenaline in future similar cases. External, and then later internal, pacing allowed for a reduction in doses of both medications (and the total cessation of Isoprenaline).

Without the pacing the underlying rhythm was asystole. Several previous case studies have described successful outcomes after prolonged cardiac arrests, and a review of 43 cases of Yew Tree poisoning found CPR was required in $26(60 \%)$ of cases (4). With this in mind, it may be beneficial to have a Lucas or similar device available, if possible, to save on staff resources, shall the patient deteriorate to cardiac arrest. Replacing the fast patches the paramedics apply with hospital parches may also be a consideration, as though they may be compatible with hospital defibrillators you are not always able to pace through the patches paramedics use.

The accidentally triggered emesis on the ICU admission covid-19 throat swab may also have helped in this case. This episode occurred about 9 hours post ingestion of the seeds, and the regurgitation of whole and partially digested seeds in the vomit may well have reduced subsequent plasma levels of the taxine alkaloids and prevented any further deterioration over the following days. Toxbase does suggest initiating vomiting or using activated charcoal, but it was not deemed appropriate in the initial management due to the fluctuating GCS and possible aspiration risk. In future, earlier consideration of gastric emptying could be considered.

Poisoning from Yew Tree seeds may become more prevalent, due to the easy access to the toxic substance from online shops. We were lucky our patient informed us of what he took, allowing the team to prepare for his arrival adequately. Many patients who attempt suicide through ingestion of substances may not share this information, and so supportive therapy and treating symptoms may be the best option. In this case, there was no direct antidote and so supportive therapy was also all that was available. A successful outcome was obtained without the need for ECMO by following the valuable guidelines of Toxbase, verbal conversations with UK NPIS and the experience of the multi-disciplinary acute care team. Whilst this case was managed successfully in a district general hospital we would still recommend early discussion with UK NPIS and the local ECMO centre, as early transfer to a more appropriate centre may be required.

\section{Authorship}

Author 1: Treated the patient, collated the information, researched the case and wrote the report

Author 2: Treated the patient, collated the information and reviewed and approved of the report

\section{Acknowledgements}

The authors would like to acknowledge Dr Shanmugam and Dr Raghavan, South Warwickshire Foundation Trust, for their assistance.

Published with written consent of the patient and their parent.

\section{Conflict of Interest}

No external funding and no competing interests declared.

\section{References}

1: The Woodland Trust. Yew (Taxus Baccata) https://www.woodlandtrust.org.uk/trees-woods-andwildlife/british-trees/a-z-of-british-trees/yew/ (Accessed 11/11/2021)

2: Wilson CR, Sauer JM, Hooser SB. Taxines: a review of the mechanism and toxicity of yew (Taxus spp.) alkaloids. Toxicon . 2001;39 : 175-85.

3: Piskač O, Stříbrný J, Rakovcová H, Malý M. Cardiotoxicity of yew.Cor et Vasa . 2015; 57 : 234-8. 
4: Alarfaj M, Goswami A. Cardiotoxicity in yew berry poisoning: a case report and literature review. The American Journal of Emergency Medicine . 2021; 21 : 00426-5.

5: Van Ingen G, Visser R, Peltenburg H, Van Der Ark AM, Voortman M. Sudden unexpected death due to Taxus poisoning. A report of five cases, with review of the literature. Forensic science international.1992; $56: 81-7$.

6: Toxbase (2016) Taxus baccata (Yew), 2016. https://www.toxbase.org/poisons-index-a-z/y-products/yew/ (Accessed 11/11/2021)

\section{Figure 1}

Admission ECG showing a ventricular rhythm with a prolonged QT (QT 670ms / QTc 414ms).

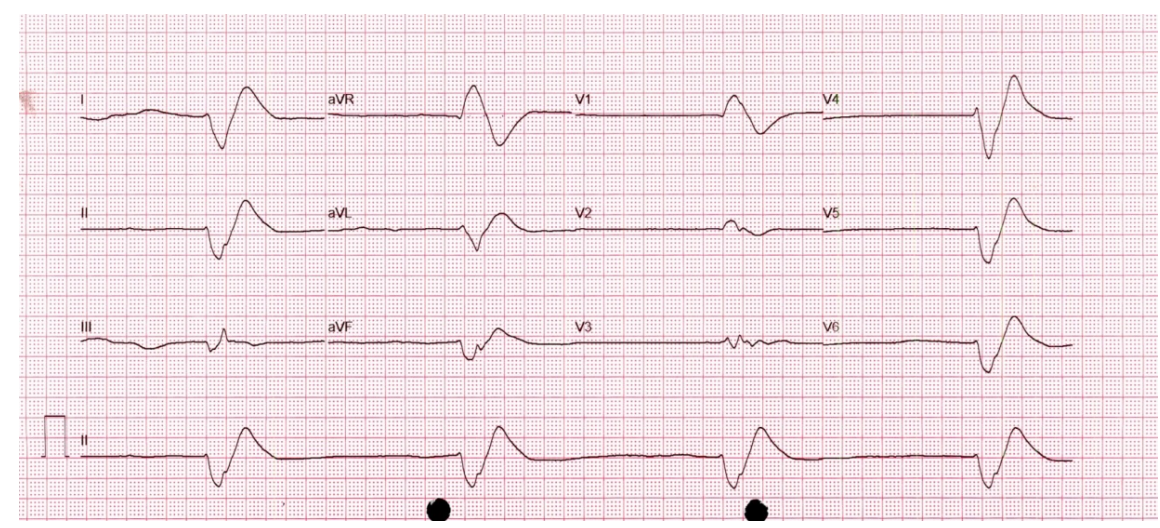

\section{Table 1}

Arterial blood gas results in the first 90 minutes after admission to the Emergency Department.

\begin{tabular}{llllll}
\hline Time & $\mathrm{pH}$ & $\mathrm{HCO} 3$ & Lactate & Glucose & $\mathrm{K}$ \\
\hline 03:08 & 7.19 & 24.8 & 3.5 & 8.7 & 3.8 \\
$03: 47$ & 7.35 & 16.6 & 3.1 & 14.9 & 3.6 \\
04:30 & 7.46 & 15.6 & 3.4 & 16.6 & 3.1 \\
\hline
\end{tabular}

